\title{
Green discoloration of the crown after internal root resorption treatment with grey mineral trioxide aggregate (MTA).
}

\author{
Juan José Segura-Egea ${ }^{1}$, Lizett Castellanos-Cosano ${ }^{2}$, Jennifer Martín-González ${ }^{2}$, Luis Oscar Alonso- \\ Ezpeleta $^{3}$, F. Javier López Frías ${ }^{4}$
}

\footnotetext{
${ }^{1}$ Professor, Department of Endodontics, School of Dentistry, University of Sevilla, C/ Avicena s/n, 41009-Sevilla, Spain. ${ }^{2}$ Doctoral fellow, Department of Endodontics, School of Dentistry, University of Sevilla, C/ Avicena s/n, 41009-Sevilla, Spain.

${ }^{3}$ Assistant Professor, Department of Endodontics, School of Dentistry, University of Zaragoza, 22006-Huesca, Spain.

${ }^{4}$ Associate Professor, Department of Endodontics, School of Dentistry, University of Sevilla, C/ Avicena s/n, 41009-Sevilla, Spain.
}

Correspondence:

Dpto. de Estomatología,

Facultad de Odontología,

C/ Avicena s $/ n$,

41009-Seville (SPAIN)

e-mail:segurajj@us.es

\author{
Segura-Egea JJ, Castellanos-Cosano L, Martín-González J, Alonso-Ezpe- \\ leta LO, López Frías FJ. Green discoloration of the crown after internal \\ root resorption treatment with grey mineral trioxide aggregate (MTA). J \\ Clin Exp Dent. 2011;3(Supp11):e404-7. \\ http://www.medicinaoral.com/odo/volumenes/v3iSuppl1/jcedv3iSu- \\ ppl1p404.pdf
}

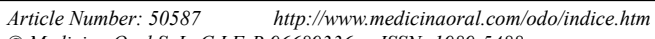

\begin{abstract}
Root resorption (RR) is the loss of dental hard tissues as a result of clastic activities. Internal inflammatory root resorption (IRR) is a type of RR characterized by progressive loss of tooth substance starting from the root canal wall. IRR is usually asymptomatic, slowly progressing, and detectable upon routine radiographic examination or by the clinical sign of a 'pink spot' when the IRR involves the crown or the coronal third of the root canal. Mineral trioxide aggregate (MTA) is a biocompatible cement that has been used successfully in pulp capping, pulpotomy, treatment of traumatized teeth with immature apices, and for treatment of root resorption. The treatment and follow-up of a maxillary central incisor with perforating IRR managed by root canal treatment and grey MTA repair is reported. IRR affected tooth 11 (FDI World Dental Federation) in a 29-year-old female patient, with a history of previous trauma. Follow-up radiographs over 24 months demonstrated the maintenance of a functional tooth. However, the tooth showed a green discoloration of the crown after MTA treatment.
\end{abstract}

Key words: Internal root resorption, Mineral trioxide aggregate, Root canal treatment. 


\section{Introduction}

Root resorption (RR) is the loss of dental hard tissues as a result of clastic activities (1). RR might be broadly classified into external or internal resorption by the location of the resorption in relation to the root surface. Internal inflammatory root resorption (IRR) is characterized by progressive loss of tooth substance starting from the root canal wall (2). IRR is usually asymptomatic, slowly progressing, and detectable upon routine radiographic examination or by the clinical sign of a 'pink spot' when the IRR involves the crown or the coronal third of the root canal (3).

Various etiologic factors have been implicated, including trauma, caries and periodontal infections, excessive heat generated during restorative procedures on vital teeth, calcium hydroxide procedures, vital root resections, anachoresis, orthodontic treatment, cracked teeth, or simply idiopathic dystrophic changes within normal pulps $(2,4)$.

For IRR to take place, vital pulp tissue is required (5). Therefore, nonsurgical root canal therapy (pulp removal) is the treatment of choice to arrest the destructive process. Treatment of IRR must aim at complete removal of the resorptive tissue from the root canal system, in an attempt to prevent further loss of hard tissue. If the process has extended to the point that it reaches the external root surface, root integrity is lost and destruction of the adjacent periodontal tissues may occur (6). Selecting suitable restorative materials for these cases remain a challenge, especially if tooth loss is extensive; extraction is the only realistic option in some cases (5). Mineral trioxide aggregate (MTA) is a biocompatible cement, ideal as orthograde or retrograde filling material (7), that has been used successfully in pulp capping, pulpotomy, treatment of traumatized teeth with immature apices, and for treatment of root resorptions (5, 6). MTA has good sealing properties, biocompatibility, bactericidal effects, radiopacity, and ability to set in the presence of blood.

This case report describes the 24-month follow-up of perforating internal root resorption in a maxillary central incisor, where gray MTA were employed to restore function.

\section{Case Report}

A 29-year-old white woman because the presence of discoloration of the dental crown of the maxillary right central incisor (11, FDI World Dental Federation) with vague pain. The medical history was non-contributory. She had a history of trauma affecting both maxillary central incisors twenty years ago. Clinical examination showed the presence of a sinus tract stoma on the buccal gingival mucosa (Fig. 1A). Radiographic examination revealed a well-circumscribed, fairly oval radiolucency in the middle third of the root (Fig. 1B). No periapical

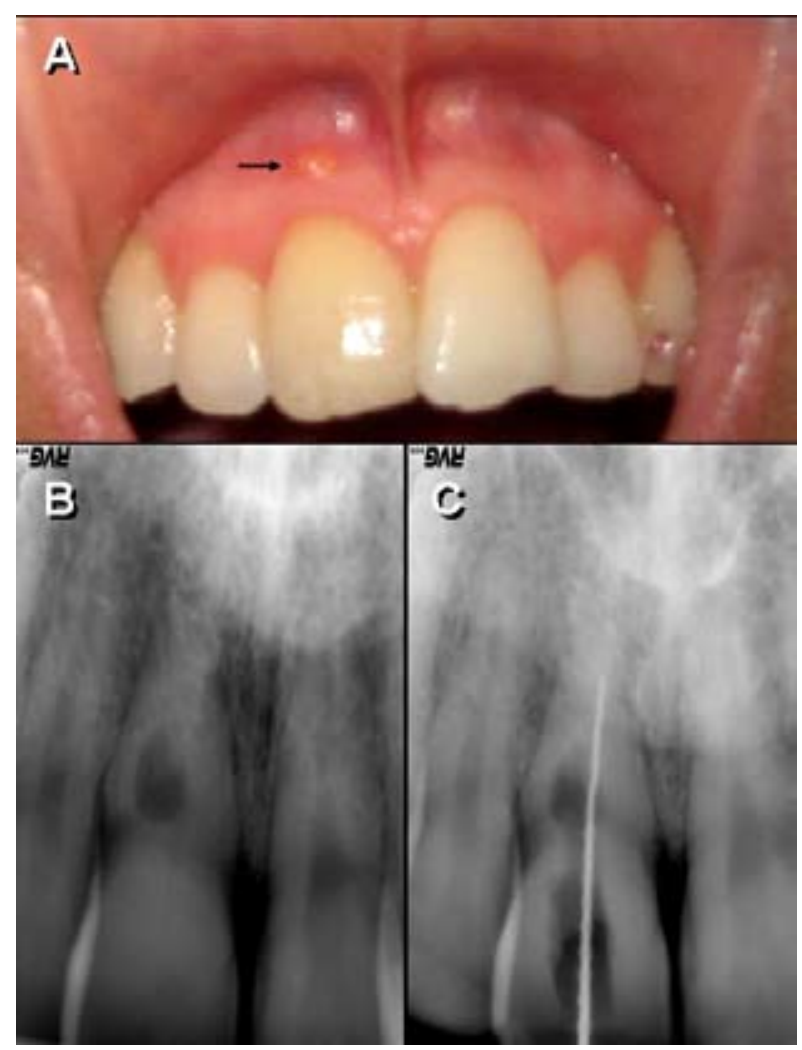

Fig. 1. (A) Clinical examination showed discoloration of the dental crown of the maxillary right central incisor (11) and the presence of a sinus tract stoma on the buccal gingival mucosa. (B) Preoperative radiograph showing a well-circumscribed, fairly oval radiolucency in the middle third of the root. $(C)$ Length determination was performed electronically and confirmed radiographically.

radiolucent lesion was detected. The electric and heat (heated gutta-percha) pulp tests were negatives. Periodontal probing depths were physiological $(<3 \mathrm{~mm})$ at all sites. Based on the clinical and radiographic findings, the diagnosis was irreversible pulpitis with perforating internal root resorption that had caused an abscess draining through the fistulous tract.

The possibility of conserving tooth 11 was considered through a combination of treatments: non surgical root canal therapy to remove the necrotic pulp and disinfect the root canal system and surgical treatment to expose the resorptive defect and to fill it up with MTA. However, patient did not agree with surgical treatment. Thus, consent was only obtained for non surgical root canal treatment.

Anaesthesia was administered accordingly, and the tooth was isolated with a rubber dam. The access cavity was opened on the palatal surface and the pulp tissue was removed. Length determination was performed electronically using the DentaPort ZX (J. Morita, Kyoto, Japan) and confirmed radiographically (Fig. 1C). During instrumentation a communication between the resorption cavity and the buccal periodontium was observed as a 


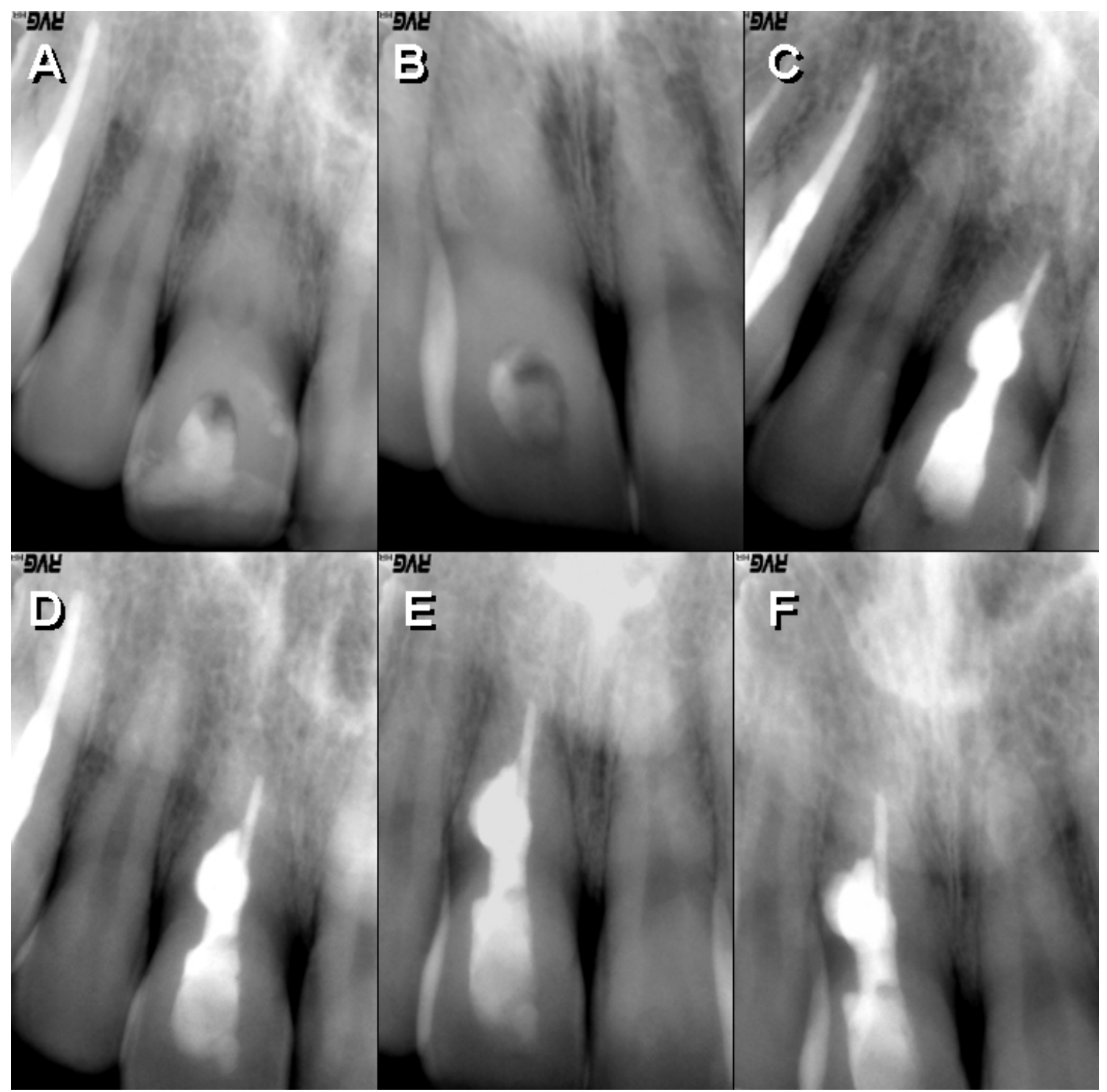

Fig. 2. (A) Periapical radiograph after filling with calcium hydroxide paste. (B) Calcium hydroxide paste remained filling the rootcanal after 30 days. (C) Immediate postoperative radiograph after MTA filling of the resorptive defect. (D) Periapical radiograph after 12 months. (E) Periapical radiograph after 18 months. (F) Periapical radiograph showing satisfactory healing after 24 months

bleeding point, in connexion with the fistulous tract. The canal was prepared by the X-Smart (Dentsply Maillefer, Ballaigues, Switzerland), which was cleaned and shaped up to an F1 instrument of the Protaper System (Dentsply-Maillefer, Ballaigues-Switzerland). An apical stop was created with a rotary nickel-titanium size 35.06 K3 (SybronEndo Dental Specialties, Orange, CA). Intracanal irrigation was performed with $5.25 \%$ $\mathrm{NaOCl}$ (Farmacia Del Campo, Madrid, Spain). Then, calcium hydroxide paste (Calcipulpe, Septodont, Saint Maur des Fosses, France) was placed to alkalinize the environment, remove remaining pulp tissue and control bleeding at the perforation and to assure the complete elimination of the granulation tissue (Fig. 2A).

After 30 days, sinus tract stoma on the buccal gingival mucosa had disappeared. Calcium hydroxide paste re- mained filling the root-canal (Fig. 2B). The dressing material was removed with $5.25 \%$ sodium hypochlorite irrigation and the root canal was dried with paper points. A 35.06 autofit gutta-percha cone (Analytic Endodontics, Orange, CA) was used to fill the apical root canal. Then, gray MTA powder was mixed according to the manufacturer's instructions and placed with an MTA carrier. MTA was firmly condensed by using a plugger and wet cotton pellets. The access cavity was sealed with Cavit G (3 M ESPE, St Paul, MN). An immediate postoperative radiograph was taken, confirming satisfactory filling of the root canal and resorptive defect (Fig. 2C). After 12 and 18 months, the patient was recalled and the tooth was found to be symptom free. No percussion sensitivity was observed, and the patient had a healthy e406 
cal radiographs showed satisfactory healing of the distal radiolucent lesion in the alveolar bone (Fig. 2D and 2E). After 24 months, tooth remains asymptomatic; however a green discoloration is evident (Fig. 2F and Fig. 3).

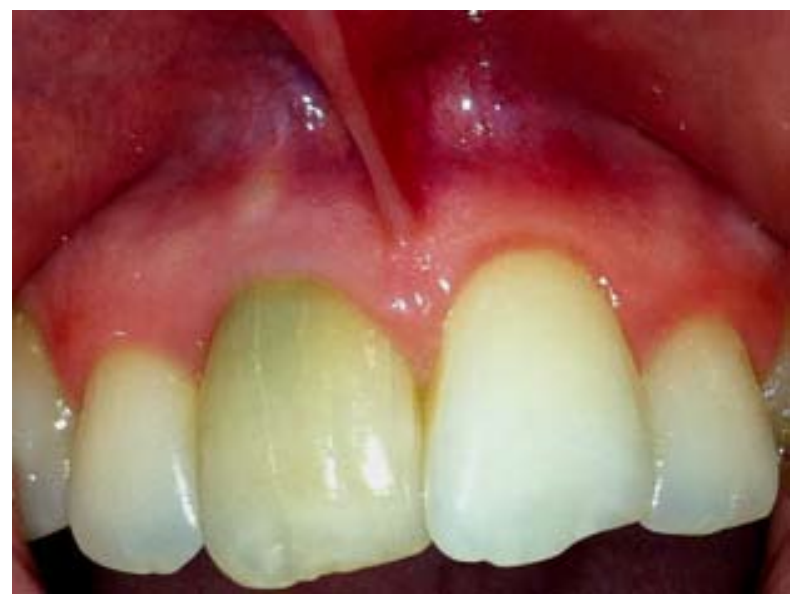

Fig. 3. After 24 months, tooth remains asymptomatic, but a green discoloration was evident.

\section{Discussion}

Internal root resorption is an insidious pathological process, initiated within the pulp space and associated with loss of dentine. It is often described as an oval shaped, symmetrically distributed over the root, enlargement of the root canal space and is usually asymptomatic and detectable by radiographs (8). The present case demonstrated a successful treatment of a perforating internal root resorption with grey MTA. Successful surgical and nonsurgical treatment of internal resorption in both primary and permanent teeth using MTA has been reported previously in several case reports $(5,9-11)$.

MTA is biocompatible (12) and has been shown to be effective in repairing furcation perforations (13) and lateral root perforations (14). The material is well-tolerated by periradicular tissues and has been shown to support almost complete regeneration of the periodontium (13). In addition, MTA has superior sealing properties when compared with other materials (15).

In the present case a hybrid technique has been used to obturate the canal. The portion of the canal apical to the resorption defect was obturated with gutta-percha, and then the resorption defect and associated perforation was sealed with grey MTA $(6,11)$.

One of the disadvantages of grey MTA is related to its colour. Discoloration of marginal gingiva after perforation repair with grey MTA has been reported (9). The potential of gingival discoloration should be considered in perforations located proximal to the marginal area. In the present case, a slight gingival discoloration was observed after 24 months, but a significant tooth crown green discoloration was evident.

In an attempt to improve this aspect, today white MTA is manufactured by Dentsply (ProRoot MTA — new colo- red formula, Tulsa, OK) and by Angelus (MTA Branco, Angelus Soluçöes Odontológicas). The major difference appears to be in the concentrations of carborundum ( $\mathrm{Al} 2 \mathrm{O} 3$ ) periclase $(\mathrm{MgO})$, and especially $\mathrm{FeO}$, with the observed values for each of these oxides being considerably lower in the white MTA than in the gray MTA. Differences in the observed $\mathrm{FeO}$ concentration are thought to be primarily responsible for the variation in colour of the white MTA in comparison with grey MTA (9). This colour change has broadened the indications for MTA, enabling its application in root perforations, pulpotomies, and direct pulp capping in anterior teeth. White MTA can be the material of choice to repair defects in which direct contact with gingival tissues or tooth crown implication is expected.

\section{References}

1. Patel S, Ricucci D, Durak C, Tay F. Internal root resorption: a review. J Endod. 2010;36:1107-21.

2. Andreasen JO, Andreasen FM. Root resorption following traumatic dental injuries. Proc Finn Dent Soc. 1992;88 (Suppl 1):95-114..

3. Gartner AH, Mack T, Somerlott RG, Walsh LC. Differential diagnosis of internal and external root resorption. J Endod. 1976;2:329-34.

4. Walton RE, Leonard LA. Cracked tooth: an etiology for 'idiopathic' internal resorption? J Endod. 1986;12:167-9.

5. Meire M, De Moor R. Mineral trioxide aggregate repair of a perforating internal resorption in a mandibular molar. J Endod. 2008;34:220-3.

6. Jacobovitz M, de Lima RKP. Treatment of inflammatory internal root resorption with mineral trioxide aggregate: a case report. Int Endod. J 2008;41:905-12.

7. Parirokh M, Torabinejad M. Trioxide Aggregate: A Comprehensive Literature Review-Part I: Chemical, Physical, and Antibacterial Properties. J Endod. 2010;36:16-27.

8. Gulabivala K, Searson LJ. Clinical diagnosis of internal resorption: an exception to the rule. Int Endod J. 1995;28:255-60.

9. Bortoluzzi EA, Aráujo GS, Guerreiro Tanomaru JM, TanomaruFilho M. Marginal gingiva discoloration by gray MTA: a case report. J Endod. 2007;33:325-7.

10. Silveira FF, Nunes E, Soares JA, Ferreira CL, Rotstein I. Double 'pink tooth' associated with extensive internal root resorption after orthodontic treatment: a case report. Dent Traumatol. 2009;25:e43-7. 11. Hsien HC, Cheng YA, Lee YL, Lan WH, Lin CP. Repair of perforating internal resorption with mineral trioxide aggregate: a case report. J Endod. 2003;29:538-9.

12. Torabinejad M, Hong CU, Pitt Ford TR, Kaiyawasam SP. Tissue reaction to implanted Super EBA and Mineral Trioxide Aggregate in the mandible of guinea pigs: a preliminary. J Endod. 1995;21:569-71. 13. Regan JD, Gutmann JL, Witherspoon DE. Comparison of Diaket and MTA when used as root-end filling materials to support regeneration of the periradicular tissues. Int Endod J. 2002;35:840-7.

14. Main C, Mirzayan N, Shabahang S, Torabinejad M. Repair of root perforations using mineral trioxide aggregate: a long term study. J Endod. 2004;30:80-3.

15. Jacobovitz M, Vianna ME, Pandolfelli VC, Oliveira IR, Rossetto HL, Gomes BP. Root canal filling with cements based on mineral aggregates: an in vitro analysis of bacterial microleakage. Oral Surg Oral Med Oral Pathol Oral Radiol Endod. 2009;108:140-4. 